\title{
Inhibition of Src family kinases overcomes anoikis resistance induced by spheroid formation and facilitates cisplatin-induced apoptosis in human mesothelioma cells
}

\author{
RYOJI EGUCHI $^{1 *}$, YUMIKO FUJITA ${ }^{2}$, CHIHARU TABATA $^{2}$, HIROYASU OGAWA $^{3}$, \\ ICHIRO WAKABAYASHI ${ }^{1}$, TAKASHI NAKANO ${ }^{2}$ and YOSHIHIRO FUJIMORI ${ }^{4 *}$ \\ Departments of ${ }^{1}$ Environmental and Preventive Medicine, ${ }^{2}$ Thoracic Oncology, ${ }^{3}$ Division of Hematology, \\ Department of Internal Medicine, ${ }^{4}$ Department of Transfusion Medicine and Cellular Therapy, \\ Hyogo College of Medicine, Nishinomiya, Hyogo 663-8501, Japan
}

Received May 22, 2015; Accepted July 2, 2015

DOI: $10.3892 /$ or.2015.4200

\begin{abstract}
Malignant mesothelioma is an aggressive tumor arising from mesothelial cells of serous membranes, and forms spheroid-like cell aggregates in pleural and peritoneal effusions. We examined the levels of anoikis, apoptosis induced by the detachment of cells from the extracellur matrix, in suspension culture in the human mesothelioma cell line NCI-H2052. NCI-H2052 cells were adherent in conventional monolayer cultures, but were found to form spheroids in suspension cultures using dishes with ultra-low cell binding capacity. NCI-H2052 cells proliferated in both cultures, but the proliferation rate was markedly lower in suspension cultures than in monolayer cultures. In addition, NCI-H2052 cells in suspension cultures showed little apoptosis, suggesting that the suspension culture induces anoikis resistance. Western blot analysis revealed that suspension cultures induced activation of Src family kinases (SFK) after spheroid formation. Dasatinib, an inhibitor of multi-tyrosine kinases including SFK, abolished anoikis resistance in suspension cultures, indicating that SFK activated by spheroid formation are responsible for anoikis resistance. Cisplatin induced apoptosis in NCI-H2052 cells, but the apoptotic rate was significantly lower in suspension cultures than in monolayer cultures, suggesting that spheroid formation is
\end{abstract}

Correspondence to: Dr Ryoji Eguchi, Department of Environmental and Preventive Medicine, Hyogo College of Medicine, Nishinomiya, Hyogo 663-8501, Japan

E-mail: r-eguchi@hyo-med.ac.jp

Professor Yoshihiro Fujimori, Department of Transfusion Medicine and Cellular Therapy, Hyogo College of Medicine, 1-1 Mukogawa-cho, Nishinomiya, Hyogo 663-8501, Japan

E-mail: fuji-y@hyo-med.ac.jp

*Contributed equally

Key words: malignant mesothelioma, suspension culture, spheroid formation, anoikis, cisplatin, Src family kinases involved in cisplatin resistance. Furthermore, a combination of dasatinib and cisplatin induced apoptosis more significantly than either alone in suspension cultures. These results suggest that spheroid formation induces resistance to anoikis and to cisplatin through SFK activation and that dasatinib facilitates cisplatin-induced apoptosis in human mesothelioma cells.

\section{Introduction}

Malignant mesothelioma is an aggressive tumor arising from mesothelial cells of serous membranes, including the pleura, peritoneum and pericardium (1-3). Mesothelioma is highly resistant to most chemotherapeutic drugs and radiation therapy (3), and surgical therapy generally show limited efficacy (3-5). So far a combination of cisplatin and pemetrexed appears to be the best chemotherapy regimen for mesothelioma, but the median survival of patients with mesothelioma remains at $<12$ months (5). Thus, new approaches for the mesothelioma treatment are urgently required.

Anoikis, a Greek word meaning 'homelessness', is defined as the subset of apoptosis triggered by cell-cell or cell-extracellular matrix (ECM) detachments $(6,7)$. Apoptosis is programmed cell death in which caspases relay messages through so-called initiator caspases to effector caspases that medicate apoptotic processes, such as externalization of phosphatidylserine, membrane blebbing and nuclear fragmentation (8). These apoptotic features are all observed during anoikis (9). Anoikis plays a crucial role in defense mechanisms by preventing the re-adhesion of detached cells to incorrect locations and their unregulated growth $(10,11)$. Anoikis resistance is emerging as a hallmark of cancer cells and contributes to invasion and metastasis formation of many types of tumors including mesothelioma (12). Three-dimensional tissue culture methods, including the formation of multicellular aggregates (spheroid) using suspension culture, have been adopted with the superiority over conventional monolayer culture to mimic the tumor behavior in vivo (13). Spheroid formation has been reported to be involved in anoikis resistance $(13,14)$. 
Src family kinases (SFK) are non-receptor and cytoplasmic tyrosine kinases that have a pivotal role in cell adhesion, proliferation, survival and apoptosis. Among the SFK, Src, Yes and Fyn show ubiquitous expression, whereas others including Lyn, exhibit more restricted tissue localization $(15,16)$. There have been many studies showing that Src protein level or its kinase activity is increased in a variety of human tumors (16). Our previous study clarified that human mesothelioma cells express Lyn in addition to Src, Yes and Fyn (17).

Mesothelioma cells form spheroid-like cell aggregates in pleural and peritoneal effusions (14). Spheroid formation in other tumor types such as osteosarcoma appears to play a role in chemoresistance (18), but whether the aggregates of mesothelioma cells are associated with chemoresistance remains unclear. In this study, we examined the association between anoikis and chemoresistance in the human mesothelioma cell line NCI-H2052. We found that suspension cultures induced spheroid formation, resulting in the development of resistance to anoikis and to cisplatin. Further clarification of cell growthrelated signal transduction revealed that suspension culture induces SFK activation and that inhibition of the SFK activation abolishes anoikis resistance, which in turn facilitates cisplatin-induced apoptosis in NCI-H2052 cells.

\section{Materials and methods}

Cell lines and reagents. A non-malignant transformed human pleural mesothelial cell line, Met5A and two human mesothelioma cell lines, NCI-H28 and NCI-H2052, were obtained from the American Type Culture Collection (Rockville, MD, USA). Conventional tissue-culture dishes (Becton-Dickinson Labware, Franklin Lakes, NJ, USA) for monolayer cultures and non-adherent dishes with ultra-low cell binding capacity (HydroCell, Cellseed Inc., Tokyo, Japan) for suspension cultures were used. Cells were cultured in culture medium composed of RPMI-1640 medium (Sigma, St. Louis, MO, USA) supplemented with $10 \%$ fetal bovine serum (Moregate, Brisbane, Australia), $5 \mu \mathrm{g} / \mathrm{ml}$ penicillin, $5 \mu \mathrm{g} / \mathrm{ml}$ streptomycin, and $10 \mu \mathrm{g} / \mathrm{ml}$ neomycin. Cells were incubated at $37^{\circ} \mathrm{C}$ under 95\% air and 5\% $\mathrm{CO}_{2}$. Cisplatin (LKT Lab., Saint Paul, MN, USA) and dasatinib (Biovision, Mountain View, CA, USA) were prepared in dimethylsulfoxide (DMSO) using the following stock solutions: $125 \mathrm{mM}$ cisplatin and $5 \mathrm{mM}$ dasatinib. DMSO was used as a vehicle control as appropriate. Other chemicals were purchased from Sigma.

Cell proliferation analysis. Cell proliferation was analyzed as described earlier with slight modifications (19). Briefly, NCI-H2052 cells were seeded at $1 \times 10^{5}$ per $5 \mathrm{ml}$ in $60-\mathrm{mm}$ dishes. The cells were incubated for 24-96 $\mathrm{h}$ and harvested by trypsinization. Cell numbers were measured with a Coulter Counter Z1 (Coulter Japan, Tokyo, Japan).

Morphological observation. Morphological observation was performed as described (20). Briefly, NCI-H2052 cells were incubated for $72 \mathrm{~h}$ as described above. For suspension cultures, the cells were spun down and the cell culture supernatant was aspirated. The cells were observed under a light microscope (Nicon, Tokyo, Japan) for phase contrast images.
Treatment with cisplatin and dasatinib. In experiments using cisplatin, cells were seeded at $2 \times 10^{5}$ per $9 \mathrm{ml}$ culture medium in $100-\mathrm{mm}$ dishes or at $2 \times 10^{3}$ per $90 \mu \mathrm{l}$ culture medium in each well of a 96-well plate, and cultured for $24 \mathrm{~h}$. After the incubation, $1 \mathrm{ml}$ and $10 \mu \mathrm{l}$ of fresh culture medium containing cisplatin or vehicle (DMSO) were added to the dishes and the wells of the plate, respectively, and incubation was continued for an additional 24-72 $\mathrm{h}$. In experiments using dasatinib, cells were seeded at $2 \times 10^{5}$ per $10 \mathrm{ml}$ culture medium containing dasatinib or DMSO in 100-mm dishes, and then cultured for 24-96 $\mathrm{h}$. The final concentration of dasatinib was chosen based on a prior study (17). In the combination experiments of cisplatin and dasatinib, cells were seeded at $2 \times 10^{5}$ per $9 \mathrm{ml}$ culture medium containing dasatinib or DMSO in $100-\mathrm{mm}$ dishes, and cultured for $24 \mathrm{~h}$. After the incubation, cisplatin or DMSO was added to $1 \mathrm{ml}$ of fresh culture medium supplemented with dasatinib or DMSO, the medium was then added to the dishes, and incubation was continued for an additional $72 \mathrm{~h}$.

Cell viability analysis. Cell viability analysis was performed as described (21). Briefly, cells were seeded in each well of a 96-well plate (Becton-Dickinson Labware) as described above. Cell viability was analyzed by a colorimetric assay using Cell Counting Kit-8 (CCK-8) (Dojin Chemical Institute, Kumamoto, Japan) according to the manufacturer's protocol. Color intensity was quantified by a microplate reader (SPECTRAmax PLUS384, Molecular Devices, Sunnyvale, CA, USA).

Flow cytometric analysis of apoptosis. Apoptosis was analyzed by flow cytometry using an Annexin V (Ax)-FITC Kit (Medical and Biological Laboratories, Nagoya, Japan) as described (21). Briefly, $1 \times 10^{5}$ cells treated with cisplatin or dasatinib were trypsinized, washed with phosphate-buffered saline (PBS) and then labeled with Ax-FITC and propidium iodide (PI). Fluorescence intensity was measured using a Cytomics FC 500 flow cytometer and CXP software (Beckman Coulter, Fullerton, CA, USA).

Western blotting and antibodies. Western blotting was performed as described (21). All antibodies used were purchased from Cell Signaling Technology (Beverly, MA, USA). All western blot analyses were performed three times and representative data are shown.

Statistical analysis. All data are presented as the means \pm standard errors (SEs) of 3-4 independent experiments. Comparisons between two groups were performed using Student's unpaired t-test $\left({ }^{*} \mathrm{p}<0.05,{ }^{* *} \mathrm{p}<0.005\right)$.

\section{Results}

NCI-H2O52 cells form spheroids and develop anoikis resistance in suspension cultures. The human mesothelioma NCI-H2052 cells adhered to the tissue-culture dish in conventional monolayer cultures (Fig. 1A, left panel), but were found to form multicellular aggregates (spheroids) in suspension cultures using a non-adherent dish with ultra-low cell binding capacity (HydroCell) after a 24-h incubation period (Fig. 1A, right panel). Anoikis, a subset of apoptosis, 
A
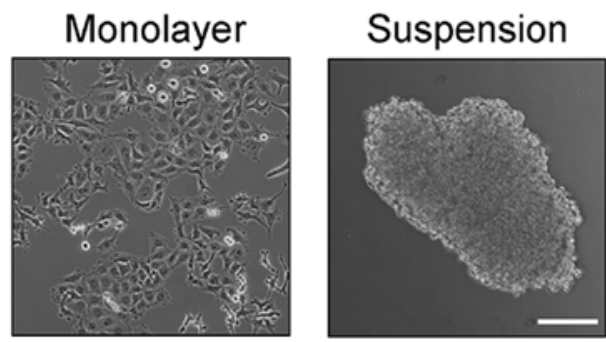

B

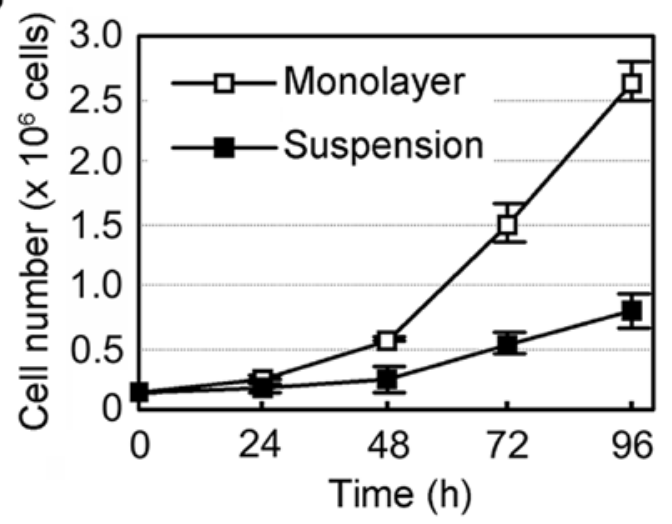

C

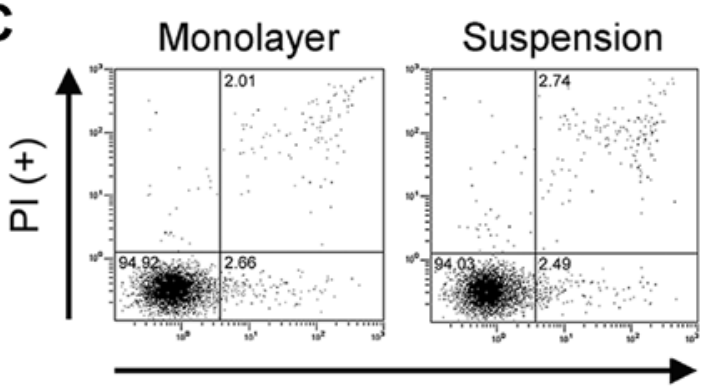

$\operatorname{Ax}(+)$

Figure 1. NCI-H2052 cells form spheroids and develop anoikis resistance in suspension cultures. (A) Phase contrast images in monolayer and suspension cultures. NCI-H2052 cells were incubated for $72 \mathrm{~h}$ as described in Materials and methods. The scale bar indicates $100 \mu \mathrm{m}$. (B) Cell proliferation assay in monolayer and suspension cultures. NCI-H2052 cell numbers were determined using a Coulter counter as described in Materials and methods. Each bar represents means \pm SEs of three independent experiments. (C) Flow cytometric analysis using double staining with Annexin V (Ax) and propidium iodide (PI). NCI-H2052 cells were incubated for $96 \mathrm{~h}$ in monolayer and suspension cultures as described in Materials and methods. The experiments in (A) and (C) were performed three times and representative data are shown.

is known to be induced by cell-cell or cell-ECM detachment and has been reported to be induced in suspension cultures of mesothelial cells (14). NCI-H2052 cells in suspension culture proliferated in a time-dependent manner, although the proliferation rate was markedly lower in suspension cultures than in monolayer cultures (Fig. 1B). Furthermore, flow cytometric analysis using double staining with Annexin V (Ax) and propidium iodide (PI) revealed that the suspension cultures as well as monolayer cultures showed little apoptosis in NCI-H2052 cells (Fig. 1C). These results suggest that suspension culture induces spheroid formation, resulting in the development of anoikis resistance in NCI-H2052 cells.

SFK activation induced by suspension culture is responsible for anoikis resistance in NCI-H2O52 cells. We examined cell
A

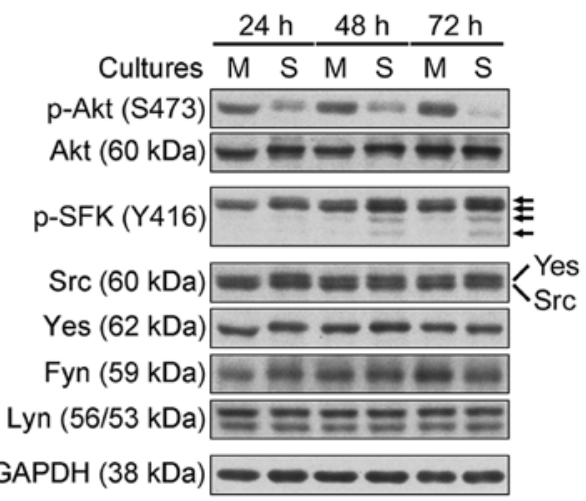

B

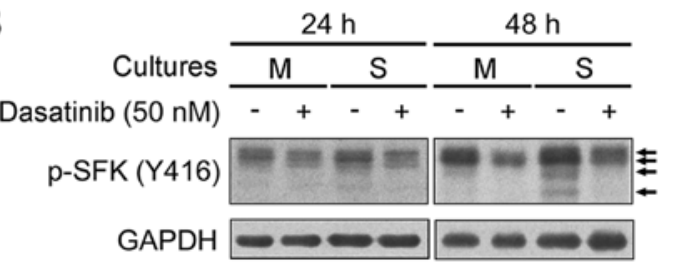

C

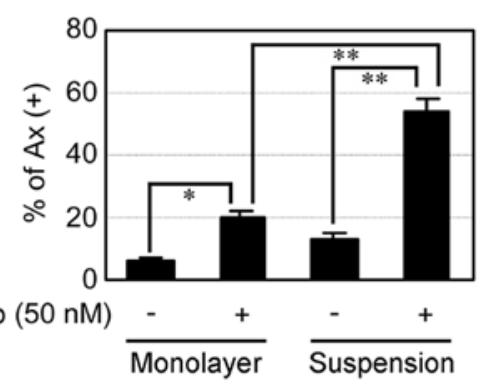

D

\begin{tabular}{rlr} 
Cultures & \multicolumn{2}{c}{$48 \mathrm{~h}$} \\
\cline { 2 - 2 } Dasatinib $(50 \mathrm{nM})$ & $-+\frac{\mathrm{S}}{-}+$ \\
Caspase-3 $(32 \mathrm{kDa})$ & \\
Cleaved Caspase-3 & \\
$(19 / 17 \mathrm{kDa})$ & \\
GAPDH &
\end{tabular}

Figure 2. SFK activated by suspension culture are involved in anoikis resistance in NCI-H2052 cells. (A) Suspension culture inactivates Akt, but activates SFK. Cell extracts were prepared from NCI-H2052 cells in monolayer and suspension cultures for 24-72 $\mathrm{h}$ as described in Materials and methods. Arrows indicate phosphorylation of Tyr-416 for various SFK. M, monolayer culture; S, suspension culture. (B) Dasatinib inhibits SFK activation in monolayer and suspension cultures. Cell extract were prepared from NCI-H2052 cells treated with $50 \mathrm{nM}$ dasatinib or vehicle (DMSO) for 24-48 h in monolayer and suspension cultures. (C) Dasatinib induces apoptosis in suspension cultures. NCI-H2052 cells were treated with $50 \mathrm{nM}$ dasatinib or DMSO for $96 \mathrm{~h}$ in monolayer and suspension cultures as described in Materials and methods. Each bar represents means \pm SEs of three independent experiments. Comparisons between two groups were performed using Student's unpaired t-test ( $\left.\mathrm{p}<0.05,{ }^{* *} \mathrm{p}<0.005\right)$. (D) Dasatinib induces caspase- 3 activation in suspension culture. Cell extracts were prepared from NCI-H2052 cells treated with $50 \mathrm{nM}$ dasatinib for $48 \mathrm{~h}$ in monolayer and suspension cultures. M, monolayer culture; S, suspension culture. The experiments in (A), (B) and (D) were performed three times and representative data are shown.

growth-related signal transduction in NCI-H2052 cells in monolayer and suspension cultures. Compared to monolayer cultures, suspension cultures suppressed Akt phosphorylation after a 24-h incubation, but induced SFK phosphorylation 


\section{A (Monolayer cultures)}

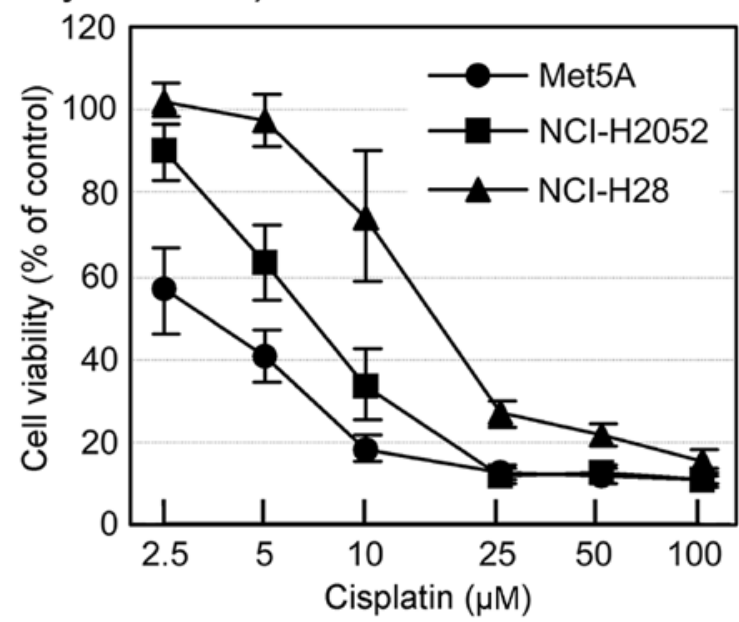

B (Monolayer cultures)

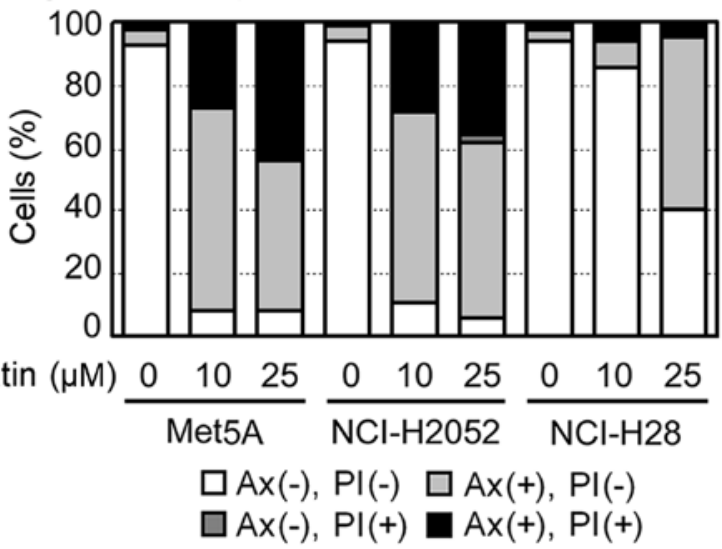

$\mathrm{C}(\mathrm{NCl}-\mathrm{H} 2052$ cells $)$

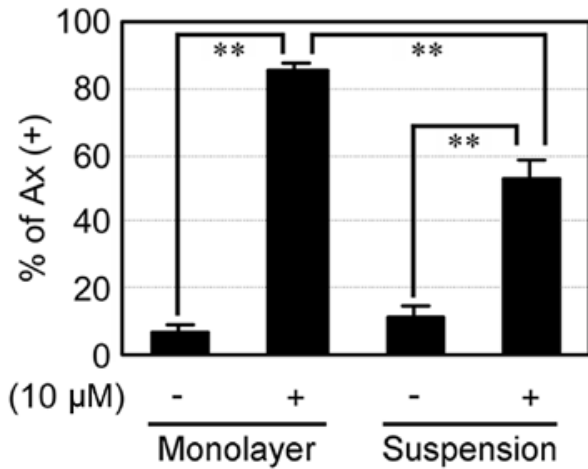

Figure 3. Cisplatin-induced apoptosis is suppressed by suspension culture in NCI-H2052 cells. (A) Cisplatin reduces the cell viability of mesothelial and mesothelioma cells in monolayer culture. Met5A, NCI-H2052 and NCI-H28 cells were treated with cisplatin at the indicated concentrations for $72 \mathrm{~h}$, and cell viability was assessed using WST- 8 as described in Materials and methods. Each bar represents means \pm SEs of three independent experiments. (B) Flow cytometric analysis using double staining with Ax and PI. Met5A, NCI-H2052 and NCI-H28 cells were treated with cisplatin at 10 and $25 \mu \mathrm{M}$ for $72 \mathrm{~h}$. Representative data of three independent experiments with similar results are shown. (C) Suspension culture suppresses cisplatin-induced apoptosis in NCI-H2052 cells. NCI-H2052 cells were treated with $10 \mu \mathrm{M}$ cisplatin or DMSO for $72 \mathrm{~h}$ in monolayer and suspension cultures. Each bar represents means \pm SEs of three independent experiments. Comparisons between two groups were performed using Student's unpaired t-test $\left({ }^{* *} \mathrm{p}<0.005\right)$.

after a 48-h incubation in a time-dependent manner (Fig. 2A). We then investigated the effects of dasatinib, an inhibitor of

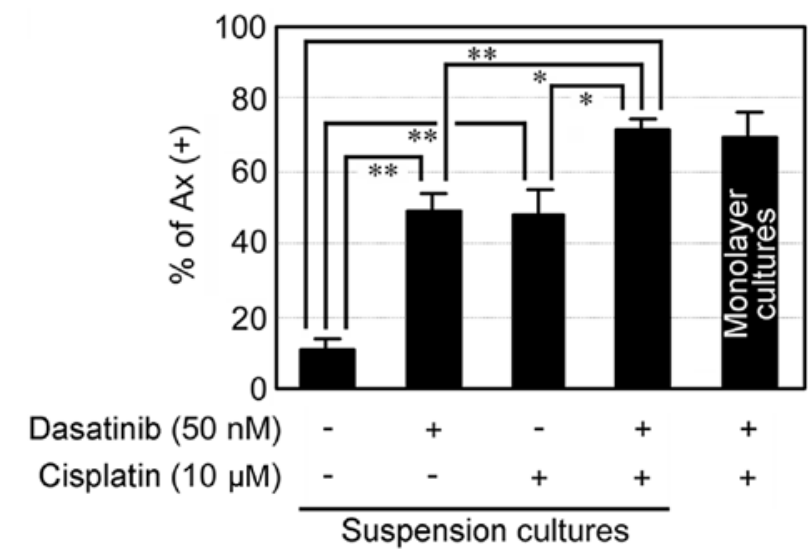

Figure 4. Inhibition of SFK activation in suspension culture negates resistance to cisplatin in NCI-H2052 cells. NCI-H2052 cells were seeded and incubated for $24 \mathrm{~h}$ in culture medium treated with $50 \mathrm{nM}$ dasatinib or DMSO in monolayer and suspension cultures. The cells were then treated with $50 \mathrm{nM}$ dasatinib or DMSO together with $10 \mathrm{nM}$ cisplatin or DMSO for $72 \mathrm{~h}$ in monolayer and suspension cultures. Each bar represents means \pm SEs of four independent experiments. Comparisons between two groups were performed using Student's unpaired t-test $\left({ }^{*} \mathrm{p}<0.05,{ }^{* *} \mathrm{p}<0.005\right)$.

multi-tyrosine kinases including SFK, on NCI-H2052 cells in monolayer and suspension cultures. Dasatinib inhibited SFK phosphorylation in monolayer and suspension cultures (Fig. 2B). Dasatinib slightly induced apoptosis in monolayer cultures, but apoptosis was markedly induced by dasatinib in suspension cultures (Fig. 2C). In addition, dasatinib markedly induced caspase-3 cleavage in suspension cultures (Fig. 2D). Thus, inhibition of SFK activation by dasatinib abolished anoikis resistance induced by spheroid formation. Collectively, these results suggest that SFK activation induced by spheroid formation is responsible for anoikis resistance in NCI-H2052 cells.

Spheroid formation in suspension cultures develops resistance to cisplatin in NCI-H2052 cells. Subsequently, we investigated the effects of cisplatin on the human mesothelial cell line Met5A, and the human mesothelioma cell lines NCI-H2052 and NCI-H28. In monolayer cultures, cisplatin reduced cell viability in Met5A and NCI-H2052 cells to a much higher degree than in NCI-H28 cells (Fig. 3A). In addition, cisplatin induced apoptosis in these cell lines in a concentration-dependent manner, but $10 \mu \mathrm{M}$ cisplatin did not induce apoptosis in NCI-H28 cells compared to Met5A and NCI-H2052 cells (Fig. 3B). These results suggest that NCI-H2052 cells as well as Met5A cells are more sensitive to cisplatin than NCI-H28 cells in monolayer cultures. Intriguingly, suspension cultures suppressed cisplatin-induced apoptosis compared to monolayer cultures in NCI-H2052 cells (Fig. 3C). These results suggest that spheroid formation in suspension culture induces cisplatin resistance in NCI-H2052 cells.

Dasatinib facilitates cisplatin-induced apoptosis in suspension culture in NCI-H2052 cells. To further investigate whether dasatinib facilitates cisplatin-induced apoptosis in suspension culture, we treated NCI-H2052 cells with dasatinib together with cisplatin. Treatment with the combination of dasatinib and cisplatin induced apoptosis more significantly 
Mesothelioma cells

Suspension

(pleural and peritoneal effusions)

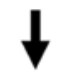

Spheroid formation

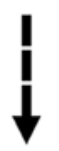

SFK activation $\longmapsto$ Dasatinib

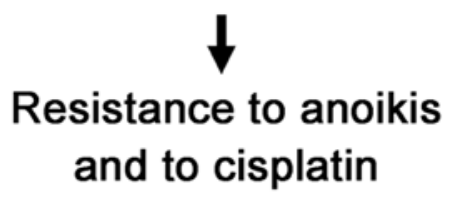

Figure 5. Proposed schematic of the mechanism of resistance to anoikis and to cisplatin in mesothelioma cells. Mesothelioma cells form spheroids in suspension similar to those observed in pleural and peritoneal effusions. The spheroids activate SFK, which leads to the developmentof resistance to anoikis and to cisplatin.

than that with either alone in suspension cultures (Fig. 4). In addition, the degree of apoptosis induced by the combination of dasatinib and cisplatin in suspension cultures was comparable to that in monolayer cultures. The results suggest that dasatinib abolishes anoikis resistance, leading to facilitation of cisplatin-induced apoptosis in spheroid-forming NCI-H2052 cells.

\section{Discussion}

As most tissues have three-dimensional structures composed of multiple types of cells, suspension culture that induces spheroid formation has been increasingly used in the field of cancer research to mimic the in vivo condition. In the present study, we adopted a suspension culture system by using a non-adherent dish with ultra-low cell binding capacity, which enabled NCI-H2052, a mesothelioma cell line, to form spheroids (Fig. 1). We further used this culture system to analyze the sensitivity of these mesothelioma cells to anoikis and druginduced apoptosis.

Anoikis is the subset of apoptosis triggered by the detachment of cells from other cells or the ECM, and anoikis resistance is associated with cancer metastasis. We found that NCI-H2052 cells in suspension cultures formed spheroids, which led to the induction of anoikis resistance (Fig. 1). Mesothelioma cells form spheroid-like cell aggregates in pleural and peritoneal effusions, and mesothelioma cell lines, but not the non-malignant mesothelial cell line Met5A, resist anoikis as multicellular aggregates in suspension culture (14). Some tumors, including lung and ovarian cancers, have shown a similar correlation between spheroid formation and anoikis resistance $(22,23)$. These results suggest that spheroid formation of malignant tumors favors the induction of anoikis resistance.

Akt is a serine/threonine kinase that plays a crucial role in multiple cellular processes such as cell proliferation, survival and apoptosis, and especially inactivates pro-apoptotic factors, leading to cell survival (24). In anoikis resistant osteosarcoma cells, Akt activity was upregulated in suspension cultures (25). However, in the present study, Akt activity in suspension cultures was lower than that in monolayer cultures (Fig. 2). Similarly, Barbone et al reported that Akt activity is downregulated in suspension cultures in mesothelioma cells (26). Presently, we have no rational explanation for this difference, but given the multifactorial nature of cancer, it is possible that Akt downregulation in anoikis resistance may be mesothelioma-specific. In addition, this Akt downregulation may be associated with the observed decrease in growth rate of NCI-H2052 cells in suspension cultures.

Malignant mesothelioma is refractory to conventional chemotherapy, which is related to resistance to the apoptosis induced by chemotherapeutic drugs. It has been reported that spheroid cells in osteosarcoma and ovarian cancer are more resistant to chemotherapeutic drugs than their parental cells $(18,27)$. In the present study, spheroid-forming NCI-H2052 cells developed cisplatin resistance (Fig. 3). Thus, it seems to be a general phenomenon that spheroid formation of malignant tumors induces chemoresistance.

$\mathrm{v}$-Src transformation in MDCK cells induces anoikis resistance (6), but how SFK including endogenous Src (c-Src) are involved in anoikis resistance remains unclear in malignant mesothelioma. It has been reported that suspension culture induces Src activation in anoikis resistant osteosarcoma cells (25). Similarly, we found that suspension cultures induced SFK activation and anoikis resistance in mesothelioma cells (Fig. 2). Our previous study also showed that the Fyn-expressing mesothelioma NCI-H2052 cells, are more insensitive to SFK inhibitors including dasatinib than Fyn-deficient mesothelioma cells, NCI-H28, in monolayer cultures (17). However, we observed that the rate of NCI-H2052 cell apoptosis induced by dasatinib in suspension cultures was more prominent than that in monolayer cultures (Fig. 2). These results suggest that inhibition of SFK activation abolishes the anoikis resistance of spheroid-forming mesothelioma cells. Furthermore, the present study shows for the first time that inhibition of SFK activation in spheroid cultures of a mesothelioma cell line facilitates cisplatin-induced apoptosis (Fig. 4). Phase II clinical trials have shown that dasatinib alone has been ineffective in unselected mesothelioma patients (28), but our study suggests that combination therapy using dasatinib and cisplatin may be more effective than dasatinib alone in the treatment of mesothelioma.

In the present study, spheroid formation in suspension cultures was observed after a 24-h incubation period (data not shown), but these suspension cultures activated SFK only after a 48-h incubation period (Fig. 3), suggesting that spheroid formation preceded SFK activation. In addition, we observed that dasatinib treatment could partially attenuate spheroid aggregation (data not shown). However, it is still uncertain whether SFK are directly involved in spheroid formation. Further studies are needed to clarify the precise relationship between spheroid formation and SFK activation.

In conclusion, mesothelioma cells form spheroids in suspension culture that induces SFK activation, resulting in developing resistance to anoikis and to cisplatin (Fig. 5). This study also suggests that the combination of dasatinib 
and cisplatin is potentially useful for treatment of malignant mesothelioma.

\section{Acknowledgements}

This study was supported by KAKENHI to Y. Fujimori, Grantin-Aid for Researchers, Hyogo College of Medicine, 2013 and MEXT-Supported Program for the Strategic Research Foundation at Private Universities, 2012-2015.

\section{References}

1. Robinson BW, Musk AW and Lake RA: Malignant mesothelioma. Lancet 366: 397-408, 2005.

2. Tsao AS, Wistuba I, Roth JA and Kindler HL: Malignant pleural mesothelioma. J Clin Oncol 27: 2081-2090, 2009.

3 . Vogelzang NJ: Chemotherapy for malignant pleural mesothelioma. Lancet 371: 1640-1642, 2008.

4. Goudar RK: New therapeutic options for mesothelioma. Curr Oncol Rep 7: 260-265, 2005.

5. Vogelzang NJ, Rusthoven JJ, Symanowski J, Denham C, Kaukel E, Ruffie P, Gatzemeier U, Boyer M,Emri S, Manegold C, et al: Phase III study of pemetrexed in combination with cisplatin versus cisplatin alone in patients with malignant pleural mesothelioma. J Clin Oncol 21: 2636-2644, 2003.

6. Frisch SM and Francis H: Disruption of epithelial cell-matrix interactions induces apoptosis. J Cell Biol 124: 619-626, 1994.

7. Meredith JE Jr, Fazeli B and Schwartz MA: The extracellular matrix as a cell survival factor. Mol Biol Cell 4: 953-961, 1993.

8. Cohen GM: Caspases: The executioners of apoptosis. Biochem J 326: 1-16, 1997.

9. Valentijn AJ, Zouq N and Gilmore AP: Anoikis. Biochem Soc Trans 32: 421-425, 2004.

10. de Ridder L, Cornelissen $\mathrm{M}$ and de Ridder D: Autologous spheroid culture: A screening tool for human brain tumour invasion. Crit Rev Oncol Hematol 36: 107-122, 2000.

11. Simpson CD, Anyiwe K and Schimmer AD: Anoikis resistance and tumor metastasis. Cancer Lett 272: 177-185, 2008.

12. Zhong X and Rescorla FJ: Cell surface adhesion molecules and adhesion-initiated signaling: Understanding of anoikis resistance mechanisms and therapeutic opportunities. Cell Signal 24 393-401, 2012.

13. Mueller-Klieser W: Three-dimensional cell cultures: From molecular mechanisms to clinical applications. Am J Physiol 273: C1109-C1123, 1997.

14. Daubriac J, Fleury-Feith J, Kheuang L, Galipon J, Saint-Albin A, Renier A, Giovannini M, Galateau-Sallé F and Jaurand MC: Malignant pleural mesothelioma cells resist anoikis as quiescent pluricellular aggregates. Cell Death Differ 16: 1146-1155, 2009.
15. Thomas SM and Brugge JS: Cellular functions regulated by $\mathrm{Src}$ family kinases. Annu Rev Cell Dev Biol 13: 513-609, 1997.

16. Yeatman TJ: A renaissance for SRC. Nat Rev Cancer 4: 470-480, 2004.

17. Eguchi R, Kubo S, Takeda H, Ohta T, Tabata C, Ogawa H, Nakano T and Fujimori Y: Deficiency of Fyn protein is prerequisite for apoptosis induced by Src family kinase inhibitors in human mesothelioma cells. Carcinogenesis 33: 969-975, 2012.

18. Arai K, Sakamoto R, Kubota D and Kondo T: Proteomic approach toward molecular backgrounds of drug resistance of osteosarcoma cells in spheroid culture system. Proteomics 13: 2351-2360, 2013.

19. Eguchi R, Kubo S, Ohta T, Kunimasa K, Okada M, Tamaki H, Kaji K, Wakabayashi I, Fujimori Y and Ogawa H: FK506 induces endothelial dysfunction through attenuation of Akt and ERK1/2 independently of calcineurin inhibition and the caspase pathway. Cell Signal 25: 1731-1738, 2013.

20. Eguchi R, Fujimori Y, Ohta T, Kunimasa K and Nakano T: Calpain is involved in cisplatin-induced endothelial injury in an in vitro three-dimensional blood vessel model. Int J Oncol 37: 1289-1296, 2010.

21. Eguchi R, Fujimori Y, Takeda H, Tabata C, Ohta T, Kuribayashi K, Fukuoka $\mathrm{K}$ and Nakano T: Arsenic trioxide induces apoptosis through JNK and ERK in human mesothelioma cells. J Cell Physiol 226: 762-768, 2011.

22. Carduner L, Picot CR, Leroy-Dudal J, Blay L, Kellouche S and Carreiras F: Cell cycle arrest or survival signaling through $\alpha \mathrm{v}$ integrins, activation of PKC and ERK1/2 lead to anoikis resistance of ovarian cancer spheroids. Exp Cell Res 320: 329-342, 2014.

23. McCarroll JA, Gan PP, Erlich RB, Liu M, Dwarte T, Sagnella SS, Akerfeldt MC, Yang L, Parker AL, Chang MH, et al: TUBB3/ $/ \mathrm{III}-$ tubulin acts through the PTEN/AKT signaling axis to promote tumorigenesis and anoikis resistance in non-small cell lung cancer. Cancer Res 75: 415-425, 2015.

24. Datta SR, Brunet A and Greenberg ME: Cellular survival: A play in three Akts. Genes Dev 13: 2905-2927, 1999.

25. Diaz-Montero CM, Wygant JN and McIntyre BW: PI3-K/ Akt-mediated anoikis resistance of human osteosarcoma cells requires Src activation. Eur J Cancer 42: 1491-1500, 2006.

26. Barbone D, Yang TM, Morgan JR, Gaudino G and Broaddus VC: Mammalian target of rapamycin contributes to the acquired apoptotic resistance of human mesothelioma multicellular spheroids. J Biol Chem 283: 13021-13030, 2008.

27. Liao J, Qian F, Tchabo N, Mhawech-Fauceglia P, Beck A, Qian Z, Wang X, Huss WJ, Lele SB, Morrison CD, et al: Ovarian cancer spheroid cells with stem cell-like properties contribute to tumor generation, metastasis and chemotherapy resistance through hypoxia-resistant metabolism. PLoS One 9: e84941, 2014.

28. Dudek AZ, Pang H, Kratzke RA, Otterson GA, Hodgson L, Vokes EE, Kindler HL; Cancer and Leukemia Group B: Phase II study of dasatinib in patients with previously treated malignant mesothelioma (cancer and leukemia group B 30601): a brief report. J Thorac Oncol 7: 755-759, 2012. 